\title{
BMJ Open Control Crohn Safe with episodic adalimumab monotherapy as first-line treatment study (CoCroS): study protocol for a randomised controlled trial
}

\author{
Laura Janssen (1D ,1,2 Mariëlle Romberg-Camps, ${ }^{3}$ Ad van Bodegraven, ${ }^{3}$ \\ Jeoffrey Haans, ${ }^{2}$ Michèl Aquarius, ${ }^{4}$ Paul Boekema, ${ }^{5}$ Tamara Munnecom, ${ }^{6}$ \\ Lloyd Brandts, ${ }^{7}$ Manuela Joore, ${ }^{7,8}$ Adrian Masclee, ${ }^{2}$ D Jonkers, ${ }^{1}$ M Pierik ${ }^{2}$
}

To cite: Janssen L, RombergCamps M, van Bodegraven A, et al. Control Crohn Safe with episodic adalimumab monotherapy as first-line treatment study (CoCroS): study protocol for a randomised controlled trial. BMJ Open 2021;11:e042885. doi:10.1136/ bmjopen-2020-042885

- Prepublication history and supplemental material for this paper is available online. To view these files, please visit the journal online (http://dx.doi. org/10.1136/bmjopen-2020042885).

Received 17 July 2020 Revised 15 December 2020 Accepted 11 April 2021

\section{Check for updates}

(c) Author(s) (or their employer(s)) 2021. Re-use permitted under CC BY-NC. No commercial re-use. See rights and permissions. Published by BMJ.

For numbered affiliations see end of article.

Correspondence to Laura Janssen;

laura.janssen@

maastrichtuniversity.nl

\section{ABSTRACT}

Introduction Crohn's disease (CD) is a chronic inflammatory bowel disease with a heterogeneous clinical presentation, relapse rate and treatment response. At present, no markers are available to adequately predict disease course at diagnosis. To prevent overtreatment of patients with a relative mild disease course, a step-up approach starting with corticosteroids is usually applied. Timely introduction of potentially disease modifying drugs and tight control of mucosal inflammation are crucial to prevent disease-related complications in patients with a complex disease course. We hypothesise that episodic treatment with adalimumab monotherapy in combination with close monitoring after drug discontinuation improves long-term outcome and reduces drug-related side effects, while preventing overtreatment.

Methods and analysis In this pragmatic multicentre randomised controlled trial, newly diagnosed CD patients or CD patients with a flare, naïve to thiopurines and biologicals, will be included and randomised 1:1 to openlabel episodic (ie, 24 weeks) adalimumab monotherapy or step-up care starting with corticosteroids. The primary outcome is the number of yearly quarters of corticosteroid free clinical (Monitor Inflammatory Bowel Disease At Home score $\leq 3$ ) and biochemical (C reactive protein within normal range and faecal calprotectin $\leq 200 \mu \mathrm{g} / \mathrm{g}$ ) remission at week 96 . Secondary outcomes are total healthcare costs, cumulative corticosteroid dose, proportion of patients with endoscopic remission at week 24, corticosteroid-free clinical remission, time to remission and patient-reported outcome measures on quality of life, (work) disability and treatment adherence. Safety outcomes are drugrelated and disease-related adverse events and disease progression on MRI-enterography at week 96 .

Ethics and dissemination This study is approved by the Medical Research Ethics Committee of azM/UM in Maastricht dated 21 August 2019 (METC18-076) and is monitored by the Clinical Trial Centre Maastricht according to Good Clinical Practice guidelines. Written informed consent will be obtained from all patients. Study results will be published in international peer-reviewed medical journals.

\section{Strengths and limitations of this study}

- First randomised controlled trial evaluating efficacy and safety of adalimumab monotherapy as first-line treatment in Crohn's disease, alongside health economic analyses.

- The study design is pragmatic, so the results can be extrapolated to real-life practice of Crohn's disease and have high external validity.

- Patients are tightly home monitored by telemedicine tool mylBDcoach and faecal calprotectine tests, ensuring timely adjustment of treatment if necessary.

- Treatment allocation and data analyses are not blinded.

Trial registration number NCT03917303.

\section{INTRODUCTION}

Crohn's disease (CD) and ulcerative colitis are debilitating diseases characterised by a chronic relapsing and remitting course, together referred to as inflammatory bowel disease (IBD). In the Netherlands, at present over 80000 residents suffer from IBD and the incidence is increasing. ${ }^{1}$ Direct healthcare costs for IBD exceed $€ 300 \mathrm{M}$ a year in the Netherlands, of which medication cost is the major cost driver. ${ }^{2}$

The complex and multifactorial aetiology of $\mathrm{CD}$ results in a highly heterogeneous clinical presentation, relapse rate and treatment response. Insufficient control of mucosal inflammation, due to treatment delay or insufficient monitoring, leads to irreversible bowel damage and complications, such as stenosis and fistula. ${ }^{3}$ There is no curative treatment and no markers exist to adequately predict the disease course or treatment response at diagnosis. 
Although there are indications that treatment and tight monitoring improve outcome over time,${ }^{34}$ the burden of CD is still increasing. ${ }^{5}$ Currently, a step-up treatment approach is used to prevent overtreatment of patients with a mild disease course and to minimise use of costly medication, such as tumour necrosis factor blockers (TNF blockers) ${ }^{67}$ Initial induction of remission using corticosteroids is followed by maintenance of remission using thiopurines. In case of insufficient treatment response, TNF blockers are introduced for re-induction and maintenance of remission, either as monotherapy or combined with thiopurine. ${ }^{6}$ However, studies have shown that this step-up approach may be unfavourable, with frequent drug-related side effects, disease progression, development of complications, chronic corticosteroid use and disability. ${ }^{28}$

The landmark step-up/top-down study pointed to an improved endoscopic outcome and reduced use of methylprednisolone with early introduction of infliximab in combination with a thiopurine. ${ }^{9}$ TNF blockers are more effective in maintaining corticosteroid free clinical and endoscopic remission when introduced within twoyears after diagnosis. ${ }^{10-13}$ In addition, drug-related adverse events occur less frequent with TNF blockers compared with corticosteroids and thiopurines. ${ }^{14-16}$ Thus, a step-up approach delays the initiation of potentially disease modifying TNF blockers in high-risk patients and prolongs poorly tolerated corticosteroid exposure.

However, early introduction of TNF blockers is no standard care. The chimeric TNF blocker infliximab has shown to be more effective in combination with a thiopurine compared with monotherapy, but combination therapy is associated with an increased risk of infections and malignancies. ${ }^{10} 17$ Additionally, the risk of antidrug antibody formation with allergic reactions and lower therapeutic response rates after stopping and reinitiation is a relative contra-indication for monotherapy or intermittent treatment with infliximab. ${ }^{10}$ Infliximab monotherapy has not been studied as first line treatment.

Adalimumab is not a chimeric but humanised monoclonal antibody with a low risk of antidrug antibody formation; hence, addition of thiopurine to adalimumab has only a marginal effect. ${ }^{12}{ }^{19}$ Moreover, combination therapy with adalimumab is not associated with a better clinical response. ${ }^{12} 19$ Therefore, we hypothesise that episodic adalimumab monotherapy as first-line treatment for $\mathrm{CD}$ in combination with close disease activity monitoring after drug discontinuation improves long-term outcome and reduces drug-related side effects, while still preventing overtreatment. In addition, we postulate that the initial additional medication costs of adalimumab will be offset by substantial gain in health and reduction in costs later on, an economic consideration that is strengthened by the recent, vast drug costs reductions due to the introduction of biosimilar adalimumab.

\section{METHODS AND ANALYSIS \\ Study objectives}

With this study, we will prospectively compare the longterm efficacy, safety and costs of episodic adalimumab monotherapy as first-line treatment to step-up care starting with corticosteroids in newly diagnosed CD patients or thiopurine and biological naive CD patients with a flare.

\section{Study design}

The Control Crohn Safe (CoCroS) study is a pragmatic, multicentre, randomised controlled trial with two arms and duration of 96 weeks. Patients will be randomised 1:1 to open-label adalimumab monotherapy or step-up care, and will be tightly monitored and treated according to a predefined algorithm. Figure 1 shows an overview of the study design and the assessments during follow-up. An

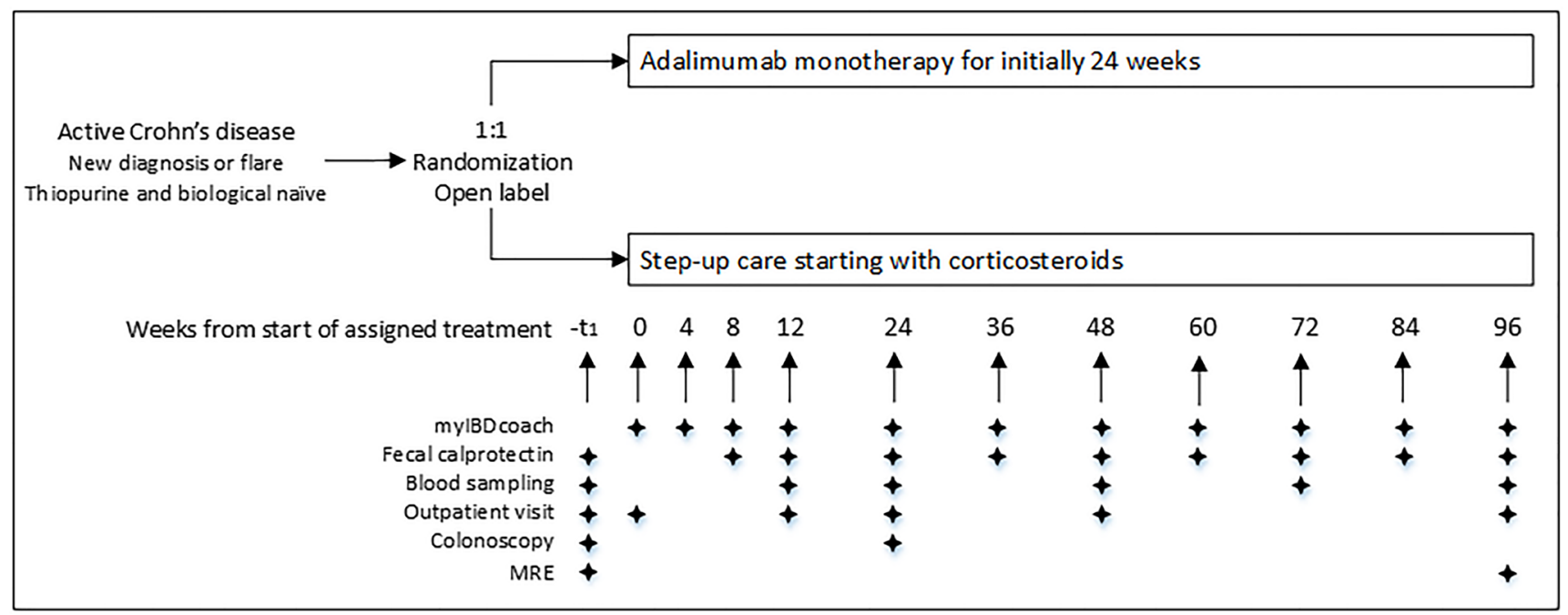

Figure 1 Schematic overview of the study design and the follow-up procedures. Blood sampling includes $C$ reactive protein, haemoglobin, white cell count, platelets, creatinine, alanine aminotransferase, alkaline phosphatase and gammaglutamyltranferase. MRE, MR enterography. 
additional file shows the study schedule in more detail (see online supplemental file 1: Standard Protocol Items: Recommendations for Interventional Trials (SPIRIT) table). The protocol is checked with the SPIRIT checklist (see online supplemental file 2: SPIRIT checklist).

\section{Outcome parameters}

The primary outcome is the number of yearly quarters of corticosteroid free clinical (Monitor IBD At Home $(\mathrm{MIAH})$ score $\leq 3$ ) and biochemical (C reactive protein (CRP) within normal range and faecal calprotectin $\leq 200 \mu \mathrm{g} / \mathrm{g}$ ) remission at week 96 . Secondary outcomes are total healthcare costs at week 96 , proportion of patients with endoscopic remission at week 24, cumulative corticosteroid dose at week 24, 48 and 96, corticosteroid-free clinical remission (according to the MIAH score as well as the Harvey Bradshaw Index) at week 24, 48 and 96, time to remission, and patient-reported outcome measures on quality of life, (work) disability and treatment adherence at week 24, 48 and 96. Additional safety outcomes are drugrelated and disease-related adverse events throughout the study and disease progression on periodic interval MRI enterography at week 96 .

\section{Study population}

Six hospitals in the Netherlands will enrol patients in the CoCroS study, including one academic hospital. The recruitment of patients started in November 2019 and full inclusion is expected in May 2022. The first patient was included in the Maastricht University Medical Center+on 23 December 2019. At the time of submission (17 July 2020), two hospitals are recruiting and we included nine patients in total.

Patients can be included if they meet all of the following inclusion criteria:

- Newly diagnosed CD or a flare of an established diagnosis of $\mathrm{CD}$, visiting the outpatient clinic or endoscopy ward of the participating centres.

- CDdiagnosisaccording toECCO-guidelines ${ }^{6}+$ complete $^{2}$ ileocolonoscopy (last endoscopy performed $<12$ months before screening)+complete small bowel imaging (MRI or CT enterography) at diagnosis.

- Naive to thiopurines and biologicals.

- 18 years up to 70 years old.

- Sufficient knowledge of Dutch language.

- Smartphone with internet access.

Patients will be excluded if they meet any of the following criteria:

- Use of corticosteroids for a duration longer than 4 months in the year before screening.

- Perianal fistula at screening.

- Severe disease requiring hospitalisation at screening.

- Short bowel syndrome or an ostomy.

- Malignancy in the past 5 years (except adequately treated non-melanoma skin cancer).

- Contraindication for TNF-blockers or immunosuppressive agents (ie, a symptomatic stricture, an abscess, a history of inadequately treated tuberculosis or other granulomatous infection, a positive chest radiograph or Quantiferon or tuberculin skin test with purified protein derivative, a recent history of an opportunistic infection (within the previous 6 months), active or ongoing infection with hepatitis $\mathrm{B}$ or $\mathrm{C}$, infection with the HIV, or multiple sclerosis).

- Contraindication for MRI-enterography and CT enterography.

\section{Study outline}

Patients will be recruited at the IBD outpatient and endoscopy departments of the participating hospitals. After signing an informed consent form, eligible patients will be randomised to adalimumab monotherapy or step-up care. Patients in the adalimumab group will receive subcutaneous adalimumab $160 \mathrm{mg}$ at week $0,80 \mathrm{mg}$ at week 2 and then $40 \mathrm{mg}$ every 2 weeks up to and including week 24. Patients in the step-up group will receive oral corticosteroids (prednisone $40 \mathrm{mg}$ per day or budesonide $9 \mathrm{mg}$ per day) with tapering according to local protocol. If treatment response is insufficient, the corticosteroid course will be prolonged and oral thiopurine maintenance will be started (azathioprine $2-2.5 \mathrm{mg} / \mathrm{kg}$, mercaptopurine $50 \mathrm{mg}$ or thioguanine $20 \mathrm{mg}$ per day), with dose adjustment based on therapeutic drug monitoring or genotype. If treatment response remains insufficient after dose optimisation, induction and maintenance using TNF blockers (intravenous infliximab $5 \mathrm{mg} / \mathrm{kg}$ at week 0 , 2, 6 and then every 8 weeks or subcutaneous adalimumab $160 \mathrm{mg}$ at week $0,80 \mathrm{mg}$ at week 2 and then $40 \mathrm{mg}$ every 2 weeks) will be started. If necessary, reinduction can be combined with a corticosteroid course and maintenance with a thiopurine. Choice of immunomodulator and TNF blocker will be made by the treating gastroenterologist and patient.

Before starting medication according to the assigned study arm, the use of oral prednisone $20 \mathrm{mg}$ per day or oral budesonide $9 \mathrm{mg}$ per day for maximal two weeks is permitted. When starting with the assigned medication, this prednisone or budesonide has to be discontinued.

All procedures in the follow-up schedule are part of standard care, except for the MRI enterography at week 96. Outpatient clinic visits are planned at screening and week 12, 24, 48 and 96 after inclusion. Other visits are scheduled on indication. During these visits, the Harvey Bradshaw Index,${ }^{20}$ medication usage, physical examination, laboratory results, imaging and/or endoscopic procedures and adverse events will be recorded using an electronic case report form. The coordinating investigator will check the completeness of these forms.

All patients fill out questionnaires via the telemedicine tool myIBDcoach every four weeks up to week 12 and thereafter monthly or threemonthly depending on disease activity. ${ }^{21}{ }^{22}$ Questions regard disease activity, medication use and side effects, but also factors affecting disease, such as social support and patient reported outcome measures on quality of life, work productivity and treatment adherence. In monitoring disease activity, myIBDcoach uses the 


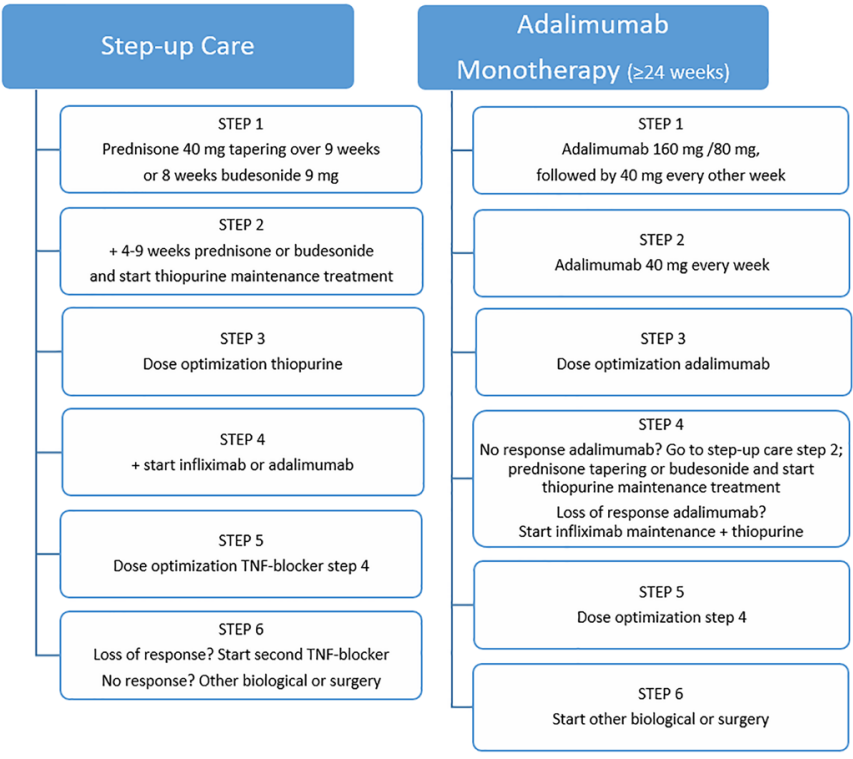

Figure 2 Schematic overview of the treatment algorithms followed in the step-up care arm and the episodic adalimumab monotherapy arm. The decision to go to the next treatment step is based on the MIAH questionnaire, faecal calprotectin, CRP and/or endoscopy. CRP, C reactive protein; MIAH, Monitor Inflammatory Bowel Disease At Home; TNF, tumour necrosis factor.

newly developed MIAH score, a symptom-based patientreported outcome measure validated relative to endoscopy ${ }^{23}$ When the disease is in remission, defined as three consecutive low monthly MIAH scores $(\leq 3)$ and faecal calprotectine scores $<200 \mu \mathrm{g} / \mathrm{g}$, patients are allowed to complete myIBDcoach questionnaires once every 3 months. Patients will measure faecal calprotectin using QuantOn Cal home tests (QoC; Preventis, Germany) at week 8 and 12, and every 12 weeks thereafter. QuantOn Cal is a faecal calprotectin immunological test combined with a smartphone application.

Patients in both study arms undergo an ileocolonoscopy in week 24 to assess mucosal healing using the simple endoscopic score for CD (SES-CD).$^{24}$ Remission is defined as a score below three and the absence of ulcers. A MRI enterography is scheduled at the end of the follow-up period to assess disease progression using the Lémann index. ${ }^{25}$

During the study period, CD specific-treatment is limited to the two study algorithms. Treatment adjustment decisions in both study arms are based on the MIAH score, CRP, faecal calprotectin and/or endoscopy. Figure 2 shows the treatment algorithms followed in both study arms. Therapeutic drug monitoring for thiopurines and TNF blockers will be performed before escalation to another drug.

Decision algorithms after endoscopic re-evaluation at week 24 are:

- SES-CD $\geq 3$ or ulcers present on colonoscopy: next treatment step.

- SES-CD $<3$ and no ulcers, MIAH score $\leq 3$, faecal calprotectine $<200 \mu \mathrm{g} / \mathrm{g}$, CRP within normal range:
- Adalimumab arm: stop adalimumab and continue monitoring.

- Step-up care-arm: continue monitoring and treatment (if initiated treatment is still ongoing).

Decision algorithms after assessment of the MIAH questionnaire, faecal calprotectine and CRP are:

- MIAH score $\leq 3$, faecal calprotectine $<200 \mu \mathrm{g} / \mathrm{g}$, CRP within normal range: continue same treatment and dose.

- Faecal calprotectin $>200 \mu \mathrm{g} / \mathrm{g}$ (no other explanation than active $\mathrm{CD}$ ): next treatment step.

- MIAH-score $>3$, faecal calprotectin $<200 \mu \mathrm{g} / \mathrm{g}$ but $>100 \%$ increase compared with previous measurement, or increase in CRP (no other explanation than active $\mathrm{CD}$ ): next treatment step or assessment of disease activity with ileocolonoscopy or imaging based on treating gastroenterologist's judgement.

- MIAH-score >3, faecal calprotectin <200 $\mu \mathrm{g} / \mathrm{g}$ and $<100 \%$ increase, normal/no increase in CRP: assessment of disease activity with ileocolonoscopy or imaging based on treating gastroenterologist's judgement.

\section{Randomisation and blinding}

After inclusion in this study, patients will be randomly assigned 1:1 to one of the two treatment arms. Randomisation will be performed centrally with the randomisation service of ALEA using the minimisation method, stratified for centre, disease location (ileal vs colon, ileocolon and upper gastrointestinal tract) and disease behaviour (inflammatory vs structuring and penetrating) at screening according to the Montreal classification. ${ }^{26}$ The minimisation method is used to balance differences between treatment groups in prognostic factors and to balance difference in initial costs of treatment per centre.

Patients, healthcare providers and staff assessing outcome measures will not be masked to treatment allocation. This open label was chosen because blinding in this treatment strategy trial with complex treatment algorithms is not feasible.

\section{Sample size}

For the primary outcome, it was estimated that 63 patients would be needed per study arm to provide an $80 \%$ power to detect a $10 \%$ increase in the number of yearly quarters of corticosteroid free remission at week 96 in the adalimumab group, assuming that the mean number in the step-up care group would be 5 with an SD of 2. This assumption is based on extrapolation of the results of previous studies. ${ }^{9}{ }^{10}$ Given the risk for nonnormally distributed data, which requires the use of a non-parametric test, the number of patients was increased with $15 \%$ to 73 in both treatment arms. Considering a loss-to-follow-up of $8 \%$, we expect inclusion of 79 patients per treatment arm is necessary.

\section{Statistical analyses}

All outcome analyses will be based on the intention to treat principle. Continuous variables will be compared 
with the t-test or Man-Whitney $U$ test depending on the distribution of the data. Categorical variables will be compared using the $\chi^{2}$ test or Fischer's exact test when necessary. Taking the total follow-up time into account, a Kaplan Meier-survival curve will be calculated and the $\log$ rank test will be used to assess the difference between treatment arms.

In addition, changes from baseline in scores for secondary outcome parameters will be analysed longitudinally using a generalised linear (mixed) model or logistic regression model as appropriate, based on the observed cases during the treatment period; treatment group, inclusion centre, period by treatment interaction and subject as fixed effects and baseline values as covariates. With regard to side effects, nature of side effects, number of events for each side effect, number of subjects with side effects and incidence will be described. Association of side effects with subject's baseline characteristics and concomitant treatment arm will be analysed using a logistic regression model.

\section{Health economic analyses}

A trial-based health economic analysis will be performed from a societal perspective with a time horizon of 96 weeks. The outcome measure for the cost-utility analysis will be the incremental costs per quality-adjusted lifeyears (QALY's) gained. The scores of patients on the EuroQol five-dimensional five-level will be converted to utility scores by using the Dutch tariff based on the preferences from a general population. ${ }^{27}$ Subsequently, QALY's are calculated by multiplying the utility score relating to a certain health state with the length of time a patient spent in that state. For the valuation of resource use, unit prices will be derived from the Pharmacotherapeutic Compass, ${ }^{28}$ the Dutch Guideline for Economic Evaluations in Healthcare and the Healthcare Insurance Board $^{29} 30$ or the hospital financial department. Productivity loss will be valued using the friction costs method. ${ }^{30}$

For the health economic analysis, data will be analysed according to an intention-to-treat principle. Since the distribution of costs is generally skewed, non-parametric bootstrap analysis will be used to quantify the uncertainty surrounding the incremental cost-effectiveness ratio (ICER). Probabilistic sensitivity analysis will be performed to estimate for example the impact of parameters on the ICER. Missing data will be imputed by using a multiple imputation approach. In addition, a budget impact analysis will be performed to quantify the financial consequences of implementing the novel studied treatment algorithm.

\section{Ethics and dissemination}

\section{Ethical and safety considerations}

The protocol has been approved by the Medical Research Ethics Committee of azM/UM in Maastricht dated 21 August 2019 (METC 2018-076). The Centrale Commissie Mensgebonden Onderzoek, as Competent Authority, has given no objection against the execution of the CoCroS study in the Netherlands. This study will be conducted in accordance with the principles of the Declaration of Helsinki and the Good Clinical Practice (GCP) guidelines and is monitored by the Clinical Trial Center Maastricht. Consent will be obtained from the participating centres and written informed consent will be obtained from all patients. An additional file shows an example of the subject information (see online supplemental file 3: English language example of subject information and consent form). All substantial changes to the protocol will be submitted as an amendment to the Medical Research Ethics Committee. The second amendment was the most recently approved, on 27 May 2020.

This study will be performed in an optimal and validated tight monitoring setting, using myIBDcoach and faecal calprotectine home tests, ensuring safety and timely adjusting or reinitiating of treatment if necessary. The risk for infectious complications and/or malignancies associated with corticosteroids, immunomodulators, biologicals and especially combination treatment, is increased in elderly patients and in children with CD. For ethical reasons, this study does not focus on these subgroups.

\section{Publication}

All study results will be published in international peer reviewed medical journals, regardless of the nature of the outcome. First and last authors will depend on the specific contributions in each manuscript. Site principal investigators will be coauthors. Study results will also be presented at relevant national and international conferences.

\section{Data deposition and curation}

Data will be coded using consecutive numbers combined with the name of the participating site. A subject identification code list will be used to link the data to the subject. The coordinating investigator, those involved in the execution of the study, research monitors and the Health and Youth Care Inspectorate have access to the source data at the investigator site. This is necessary to make sure the research is correctly performed and reliable. Patient identification log, hospital records, informed consent forms, electronic CRFs and databases are kept for 15 years after completing the study. The data are always handled confidentially, in accordance with GCP guidelines and the protocol.

\section{Patient and public involvement}

The director of the Dutch patient organisation Crohn en Colitis Ulcerosa Vereniging Nederland (CCUVN) was involved in the application for the ZonMW grant as project advisor and provided a support letter on behalf of the CCUVN. In addition, members of the structured patient collaboration programme of the IBD-clinic of MUMC +were involved in protocol development. Patients and public are not involved in recruitment or conduction of the study. The burden of intervention was assessed by representatives of patient associations participating in the Medical Research Ethics Committee. 
Patients are monitored using telemedicine tool myIBDcoach. Development of the content of myIBDcoach was a collaborative project of the Dutch patient organisation CCUVN, healthcare professionals and the IBD-section of the Dutch professional organisation of gastroenterologists. The patient-reported outcome parameters in the CoCroS study therefore reflect what patients find important in creating value.

\section{DISCUSSION}

Chronic relapsing diseases such as CD have an enormous impact on patients' quality of life, as well as on healthcare systems. Novel treatment strategies that modify the disease course and reduce the rate of relapses and complications would offer considerable benefit. As reviewed recently, well-designed trials assessing treatment strategies of early introduction of TNF blockers that prevent overtreatment and assess the disease modifying effect in combination with healthcare costs are lacking. ${ }^{11}$

The novel treatment strategy investigated in this trial aims to prevent undertreatment and overtreatment in all CD subgroups and therefore no specific group of CD is studied. Tight control of mucosal inflammation and close monitoring are important to prevent complications. ${ }^{3}$ Undertreatment and overtreatment will be prevented by assessing mucosal inflammation using colonoscopy at week 24 and if necessary adjusting treatment. In addition, patients will be tightly monitored with the frequent use of the telemedicine tool myIBDcoach and faecal calprotectin home tests.

To assess the efficacy of episodic adalimumab monotherapy as first line treatment, the primary outcome of this trial is the number of yearly quarters of corticosteroid free remission. This longitudinal primary outcome measure was chosen because it takes the entire study duration into account. In a remitting relapsing disease such as $\mathrm{CD}$, patients can experience different health states. Hence, it is important to gain information on the disease course and not just assess activity cross-sectional at a time point, especially in trials investigating long-term outcome. The goal is not only to manage active disease, but also to modify the disease course. Based on previous studies, we hypothesise that episodic treatment with adalimumab monotherapy will increase sustained remission rates compared with step-up care.

In addition, cost-utility and cost-effectiveness will be assessed in this trial. Improved long-term outcome, reduced drug-related side effects and reduced rate of hospitalisations and complications are expected to lower the costs of episodic adalimumab monotherapy. Moreover, before the start of this trial a biosimilar of adalimumab was registered in the Netherlands, resulting in a decrease of the yearly costs per patient for adalimumab from around $€ 15 \mathrm{~K}-€ 5 \mathrm{~K}$. Therefore, we hypothesise that the initial additional costs of adalimumab will be offset by substantial gain in health and reduction in costs later on and that the total direct healthcare costs between the study arms will be comparable at week 96 .

The CoCroS trial is designed to fill in a knowledge gap in the treatment (strategy) of CD. It is intended to provide insight in the (long-term) efficacy, safety and costs of early episodic adalimumab monotherapy compared with conventional step-up care. With the results, we hope to establish an evidence-based treatment strategy for CD, including improvement of long-term outcomes, improvement of quality of life, reduction of drug-related side effects, prevention of undertreatment and overtreatment and reduction of costs.

\section{Author affiliations}

${ }^{1}$ NUTRIM - School for Nutrition and Translational Research in Metabolism, Maastricht University, Maastricht, The Netherlands

${ }^{2}$ Gastroenterology and Hepatology, Maastricht UMC+, Maastricht, The Netherlands

${ }^{3}$ Gastroenterology, Zuyderland Medical Centre Sittard-Geleen, Sittard-Geleen, Limburg, The Netherlands

${ }^{4}$ Gastroenterology, VieCuri Medical Centre, Venlo, The Netherlands

${ }^{5}$ Gastroenterology, Maxima Medical Centre, Eindhoven, The Netherlands

${ }^{6}$ Gastroenterology, Laurentius Hospital, Roermond, The Netherlands

${ }^{7}$ Clinical Epidemiology and Medical Technology Assessment (KEMTA), Maastricht UMC+, Maastricht, The Netherlands

${ }^{8}$ CAPHRI - School for Care and Public Health Research Institute, Maastricht University, Maastricht, The Netherlands

Contributors MJ had the original idea for the study and drafted the initial protocol. MP led the study design and grant application. DJ, MJ, AM and AvB were project members for the grant application and contributed to the protocol development. LB and MJ planned the statistical analyses and health economic analyses. LJ, MR-C, AvB, JH, MA, PB, TM and MJ were involved in the initiation of the study at the local study sites, and are involved in the conduct of the trial. LJ led the writing of this manuscript. All other authors contributed equally with comments and feedback. All authors read and approved the final manuscript.

Funding The work is supported by ZonMW grant number 848050009 .

Competing interests None declared.

Patient consent for publication Not required.

Provenance and peer review Not commissioned; externally peer reviewed.

Supplemental material This content has been supplied by the author(s). It has not been vetted by BMJ Publishing Group Limited (BMJ) and may not have been peer-reviewed. Any opinions or recommendations discussed are solely those of the author(s) and are not endorsed by BMJ. BMJ disclaims all liability and responsibility arising from any reliance placed on the content. Where the content includes any translated material, BMJ does not warrant the accuracy and reliability of the translations (including but not limited to local regulations, clinical guidelines, terminology, drug names and drug dosages), and is not responsible for any error and/or omissions arising from translation and adaptation or otherwise.

Open access This is an open access article distributed in accordance with the Creative Commons Attribution Non Commercial (CC BY-NC 4.0) license, which permits others to distribute, remix, adapt, build upon this work non-commercially, and license their derivative works on different terms, provided the original work is properly cited, appropriate credit is given, any changes made indicated, and the use is non-commercial. See: http://creativecommons.org/licenses/by-nc/4.0/.

ORCID iD

Laura Janssen http://orcid.org/0000-0002-0414-8665

\section{REFERENCES}

1 van den Heuvel TRA, Jeuring SFG, Zeegers MP, et al. A 20-year temporal change analysis in incidence, presenting phenotype and mortality, in the Dutch IBDSL Cohort-Can diagnostic factors explain the increase in IBD incidence? J Crohns Colitis 2017;11:1169-79. 
2 van der Valk ME, Mangen M-JJ, Leenders M, et al. Healthcare costs of inflammatory bowel disease have shifted from hospitalisation and surgery towards anti-TNF $\alpha$ therapy: results from the coin study. Gut 2014:63:72-9.

3 Colombel J-F, Panaccione R, Bossuyt P, et al. Effect of tight control management on Crohn's disease (CALM): a multicentre, randomised controlled phase 3 trial. The Lancet 2017;390:2779-89.

4 Beelen EMJ, van der Woude CJ, Pierik MJ. Decreasing trends in intestinal resection and re-resection in crohn's disease: a nationwide cohort study. Ann Surg 2019;273:557-63.

5 GBD 2017 Inflammatory Bowel Disease Collaborators. The global, regional, and national burden of inflammatory bowel disease in 195 countries and territories, 1990-2017: a systematic analysis for the global burden of disease study 2017. Lancet Gastroenterol Hepatol 2020;5:17-30.

6 Gomollón F, Dignass A, Annese V, et al. 3rd European Evidencebased Consensus on the Diagnosis and Management of Crohn's Disease 2016: Part 1: Diagnosis and Medical Management. ECCOJC 2017;11:3-25.

7 Solberg IC, Cvancarova M, Vatn $\mathrm{MH}$, et al. Risk matrix for prediction of advanced disease in a population-based study of patients with Crohn's disease (the IBSEN study). Inflamm Bowel Dis 2014:20:60-8.

8 Jeuring SFG, van den Heuvel TRA, Liu LYL, et al. Improvements in the long-term outcome of Crohn's disease over the past two decades and the relation to changes in medical management: results from the population-based IBDSL cohort. Am J Gastroenterol 2017;112:325-36.

9 D'Haens G, Baert F, van Assche G, et al. Early combined immunosuppression or conventional management in patients with newly diagnosed Crohn's disease: an open randomised trial. Lancet 2008;371:660-7.

10 Colombel JF, Sandborn WJ, Reinisch W, et al. Infliximab, azathioprine, or combination therapy for Crohn's disease. $N$ Engl J Med 2010;362:1383-95.

11 Danese S, Fiorino G, Peyrin-Biroulet L. Early intervention in Crohn's disease: towards disease modification trials. Gut 2017;66:2179-87.

12 Matsumoto T, Motoya S, Watanabe K, et al. Adalimumab Monotherapy and a Combination with Azathioprine for Crohn's Disease: A Prospective, Randomized Trial. ECCOJC 2016;10:1259-66.

13 Colombel J-F, Reinisch W, Mantzaris GJ, et al. Randomised clinical trial: deep remission in biologic and immunomodulator naïve patients with Crohn's disease - a SONIC post hoc analysis. Aliment Pharmacol Ther 2015;41:734-46.

14 Goldberg R, Irving PM. Toxicity and response to thiopurines in patients with inflammatory bowel disease. Expert Rev Gastroenterol Hepatol 2015;9:891-900.
15 Lichtenstein GR, Feagan BG, Cohen RD, et al. Serious infections and mortality in association with therapies for Crohn's disease: treat registry. Clin Gastroenterol Hepatol 2006;4:621-30.

16 Steinhart AH, Ewe K, Griffiths AM. Corticosteroids for maintenance of remission in Crohn's disease. Cochrane Database Syst Rev 2003;4:Cd000301.

17 Hoentjen F, van Bodegraven AA. Safety of anti-tumor necrosis factor therapy in inflammatory bowel disease. World J Gastroenterol 2009;15:2067-73.

18 Baert F, Drobne D, Gils A, et al. Early Trough levels and antibodies to infliximab predict safety and success of reinitiation of infliximab therapy. Clin Gastroenterol Hepatol 2014;12:1474-81.

19 Kopylov U, Al-Taweel T, Yaghoobi M, et al. Adalimumab monotherapy versus combination therapy with immunomodulators in patients with Crohn's disease: a systematic review and meta-analysis. J Crohns Colitis 2014;8:1632-41.

20 Harvey RF, Bradshaw JM. A simple index of Crohn's-disease activity. Lancet 1980;1:514.

21 de Jong $\mathrm{M}$, van der Meulen-de Jong A, Romberg-Camps $\mathrm{M}$, et al. Development and feasibility study of a telemedicine tool for all patients with IBD. Inflamm Bowel Dis 2017;23:485-93.

22 de Jong MJ, van der Meulen-de Jong AE, Romberg-Camps MJ, et al. Telemedicine for management of inflammatory bowel disease (mylBDcoach): a pragmatic, multicentre, randomised controlled trial. The Lancet 2017;390:959-68.

23 de Jong MJ, Roosen D, Degens JHRJ, et al. Development and validation of a patient-reported score to screen for mucosal inflammation in inflammatory bowel disease. J Crohns Colitis 2019;13:555-63.

24 Daperno M, D'Haens G, Van Assche G, et al. Development and validation of a new, simplified endoscopic activity score for Crohn's disease: the SES-CD. Gastrointest Endosc 2004;60:505-12.

25 Pariente B, Cosnes J, Danese S, et al. Development of the Crohn's disease digestive damage score, the Lémann score. Inflamm Bowel Dis 2011;17:1415-22.

26 Silverberg MS, Satsangi J, Ahmad T, et al. Toward an integrated clinical, molecular and serological classification of inflammatory bowel disease: report of a working Party of the 2005 Montreal world Congress of gastroenterology. Can J Gastroenterol 2005;19:5A-36.

27 M Versteegh M, M Vermeulen K, M A A Evers S, et al. Dutch tariff for the five-level version of EQ-5D. Value Health 2016;19:343-52.

28 Farmacotherapeutisch Kompas, 2020. Available: http://farmacothera peutischkompas.nl

29 Health insurance board, 2020Zorginstituut Nederland. Available: www.medicijnkosten.nl

30 Zorginstituut Nederland. Richtlijn voor het uitvoeren van economische evaluaties in de gezondheidszorg, 2016. Available: https://www.zorginstituutnederland.nl 